\title{
Modelo de predicción del riesgo a corto plazo en pacientes con síndrome coronario agudo sin elevación del segmento ST
}

\section{Prediction model of short-term risk in patients with acute coronary syndrome without ST segment elevation}

\author{
Edison Muñoz, Edwin F. Arévalo, Juan Manuel SÉnior, \\ Andrés Fernández, Arturo Rodríguez, Natalia Andrea Arboleda, \\ Marta Catalina Cardona, Eliana María Arcila • Medellín (Colombia)
}

\section{Resumen}

Introducción: teniendo en cuenta los diversos factores relacionados con el pronóstico adverso en pacientes con síndrome coronario agudo sin elevación del segmento ST, es importante determinar los que se relacionan con aumento en la tasa de eventos.

Objetivo: desarrollar un modelo de predicción a corto plazo en pacientes con síndrome coronario agudo sin elevación del segmento ST, con base en las escalas TIMI y GRACE, que incluya otras variables predictoras.

Metodología: estudio observacional, analítico, de cohorte prospectiva, de desarrollo de un modelo de regresión logística, en pacientes mayores de 18 años con diagnóstico de síndrome coronario agudo sin elevación del segmento ST, que ingresan a dos centros con unidad de cuidados coronarios. Se construyeron modelos de predicción con las escalas de riesgo GRACE y TIMI como modelos independientes (modelo nulo), y comparados con un modelo de dos o tres variables formado por cada una de las escalas asociado a la creatinina y la fracción de eyección (Modelo completo). El desenlace evaluado fue el compuesto de muerte, reinfarto, ACV y sangrado.

Resultados: se recolectaron datos de 422 pacientes que ingresaron con impresión diagnóstica de SCA sin elevación del segmento ST y tuvieron seguimiento al menos durante el primer mes posterior el evento. El promedio de edad fue de $64.36 \pm 11.9$ años, el $54.1 \%$ fueron hombres y la mayoría ingresaron con diagnóstico de infarto sin elevación del segmento ST (52.7\%). La mayoría de los pacientes ingresaron en Killip I (90.8\%) y el acceso vascular para el cateterismo fue radial en el $57.7 \%$. La discriminación de los dos modelos es adecuada con estadístico C de 0.65 para TIMI y 0.69 para GRACE. La comparación de las dos curvas ROC no demuestra diferencias estadísticamente significativas ( $\mathrm{p}=0.39$ ). Los modelos completos demuestran mejor poder predictivo; sin embargo, la diferencia no es significativa. Los dos modelos finales muestran adecuada calibración (Hosmer Lemershow $\mathrm{p}=0.96$ para la escala TIMI y 0.86 para la escala GRACE).

Conclusión: en pacientes con síndrome coronario agudo sin elevación del segmento ST los modelos basados en las escalas TIMI y GRACE predicen adecuadamente el riesgo de eventos a corto plazo. (Acta Med Colomb 2015; 40: 109-117).

Palabras claves: síndrome coronario agudo, escala GRACE, escala TIMI, modelos de predicción clínica.

\section{Abstract}

Introduction: considering the various factors associated with adverse prognosis in patients with acute coronary syndrome without ST-segment elevation, is important to identify those factors associated with an increase in the rate of events.

Objective: to develop a prediction model of short-term risk in patients with acute coronary syndrome without ST segment elevation, based on the TIMI and GRACE scales, including other predictor variables.
Dres. Edison Muñoz Ortiz y Edwin F. Arévalo Guerrero: Especialistas en Medicina Interna y Cardiología, Universidad de Antioquia; Dr. Juan Manuel Sénior Sánchez: Especialista en Medicina Interna, Cardiología y Cardiología Intervencionista, Hospital Universitario San Vicente de Paúl Fundación. Coordinador Posgrado Cardiología Clínica e Intervencionista, Universidad de Antioquia; Dres. Andrés Fernández Cadavid y Arturo Rodríguez Dimuro: Especialistas en Medicina Interna, Cardiología y Cardiología Intervencionista, Hospital Universitario San Vicente de Paul Fundación; Dra. Natalia Andrea Arboleda Arias: Especialista en Medicina Interna Universidad de Antioquia; Dras. Marta Catalina Cardona Arango y Eliana María Arcila Posada: Médicas Generales, Unidad de Dolor Torácico, Hospital Universitario San Vicente de Paúl Fundación. Medellín (Colombia).

Correspondencia. Dr. Juan Manuel Sénior Sánchez. Medellín (Colombia).

E-mail: mmbt@une.net.co

Recibido: 15/X/2014 Aceptado: 23/IV2015 
Methodology: observational, analytical, prospective cohort study of development of a logistic regression model, in patients older than 18 years diagnosed with acute coronary syndrome without ST segment elevation, entering two coronary care unit centers. Prediction models were constructed with risk scales GRACE and TIMI as independent models (null model), and compared with a model of 2 or 3 variables formed by each of the scales associated with creatinine and ejection fraction (full Model ).The outcome evaluated was the composite of death, re-infarction, stroke and bleeding.

Results: data from 422 patients admitted with diagnostic impression of ACS without ST-segment elevation who were followed for at least the first month after the event, were collected. The average age was $64.36 \pm 11.9$ years. $54.1 \%$ were men and most were admitted with diagnosis of myocardial infarction without ST segment elevation (52.7\%). Most patients were admitted in Killip I (90.8\%) and vascular access for catheterization was radial at $57.7 \%$. Discrimination of the two models is adequate with $\mathrm{C}$ statistic of 0.65 for TIMI and 0.69 for GRACE. Comparison of the two ROC curves shows no statistically significant difference $(p=0.39)$. Complete models show better predictive power, but the difference is not significant. The final two models show proper calibration $(\mathrm{p}=0.96$ HosmerLemershow for the TIMI scale and 0.86 for the GRACE scale).

Conclusion: in patients with acute coronary syndrome without ST-segment elevation models based on the TIMI and GRACE scales adequately predict the risk of short-term events. (Acta Med Colomb 2015; 40: 109-117).

Keywords: acute coronary syndrome, GRACE scale, TIMI scale, clinical prediction models.

\section{Introducción}

El síndrome coronario agudo (SCA) se produce por la obstrucción del flujo sanguíneo, debido a la fractura de la placa aterosclerótica y el desencadenamiento de múltiples procesos fisiopatológicos, que llevan a un desequilibrio entre el aporte y el consumo de oxígeno, lo que induce isquemia miocárdica. Los pacientes con este síndrome, son clasificados de acuerdo con el desarrollo de alteraciones en el segmento ST en el electrocardiograma, tal como elevación para definir un grupo conocido como infarto con elevación del segmento ST, o no elevación que define al SCA sin elevación del segmento ST, entre los cuales se encuentra la angina inestable (AI) y el infarto agudo del miocardio sin elevación del segmento ST (IAMNST) (1).

Las recomendaciones de las guías de práctica clínica, enfatizan sobre el manejo oportuno de los pacientes con SCA, mediante la utilización precoz de estrategias de manejo farmacológico y de técnicas modernas de intervencionismo cardiaco (1-4). Para lograr esto se han explorado en diversos tipos de estudios algunas variables de pronóstico, pero no existe actualmente un marcador único que permita realizar con un margen aceptable de certeza esta aproximación. Ciertas características del dolor torácico se relacionan con la probabilidad de enfermedad coronaria; la presencia de los síntomas en las 48 horas previas al ingreso, se asocia con una mayor tasa de isquemia recurrente $(64 \%)$, en comparación a quienes ingresan sin angina en reposo en las últimas 48 horas (45\%), o con el patrón de angina "in crescendo" (28\%) (5). $\mathrm{El}$ electrocardiograma (EKG) es una herramienta valiosa, puesto que predice el riesgo a un año; los pacientes con EKG normales tienen un riesgo del $8 \%$ de infarto no fatal o muerte, en comparación a quienes tienen cambios aislados en la onda $\mathrm{T}$ que es $14 \%$, quienes presentan elevación del segmento ST 16\%, depresión del segmento ST $18 \%$ y a la combinación de elevación y depresión 26\% (6-8). En los últimos años, los marcadores séricos de necrosis miocárdica se han constituido en el estándar de oro para establecer el diagnóstico y el riesgo de los pacientes con SCA. Los estudios iniciales con troponina I y $\mathrm{T}$ demostraron el valor de estos marcadores $(9,10)$, el cual ha sido corroborado con métodos más sensibles (11). Otras variables han sido analizadas, sin embargo tienen un menor poder de predicción en análisis multivariado, como la fracción de eyección y el nivel de creatinina, entre otras.

La construcción de modelos multivariables de predicción de riesgo, surgieron como una necesidad para identificar aquellos pacientes que se benefician de una estrategia invasiva; entre ellos están la clasificación del ACCF/AHA (American College of Cardiology Foundation/American Heart Association) (12), el PURSUIT (13), el FRISC (14), el HEART (15) TIMI en SCA con y sin elevación del segmento ST $(16,17)$ y el GRACE $(18-20)$, entre otros. De éstos, los más utilizados y que han sido validados prospectivamente y avalados por sociedades internacionales y nacionales son el TIMI y el GRACE. La escala GRACE fue recientemente actualizada para mejorar la predicción a uno y tres años.

Teniendo en cuenta los diversos factores relacionados con el pronóstico adverso en pacientes con SCA sin elevación del segmento ST, el comportamiento diferencial del síndrome en población latina, la disponibilidad de modelos clínicos de riesgo aceptados por las guías clínicas y las posibles diferencias existentes en nuestra población, debido a factores étnicos, sociodemográficos y culturales entre otros, es importante determinar los factores que se relacionan con aumento en la tasa de desenlaces, en pacientes ingresados a dos unidades de cuidados coronarios de la 
ciudad de Medellín, con el objetivo de desarrollar un modelo predictivo, que permita establecer en forma adecuada el riesgo y por ende racionalizar los recursos, en cuanto a la necesidad de hospitalización en unidades especiales, de intervención con angioplastia e implantación de stent y la utilización de medicamentos nuevos con beneficio demostrado en población de alto riesgo.

\section{Diseño del estudio}

\section{Metodología}

Estudio observacional, analítico, de cohorte retrospectiva, de desarrollo de un modelo de regresión logística, de pacientes mayores de 18 años que ingresan a unidad especializada con diagnóstico de SCA sin elevación del segmento ST, en dos centros con unidad de cuidados coronarios y servicio de hemodinámica 24 horas. Se clasificaron los pacientes con IAMNST si tenían marcadores séricos positivos, y como AI si eran negativos. Se evaluaron dos escalas pronósticas de clasificación: la escala TIMI y GRACE; se registraron otras variables con el objetivo de construir un modelo de regresión logística, para predecir el desenlace compuesto de muerte, reinfarto, enfermedad cerebrovascular (ECV) y sangrado en los primeros 30 días del evento.

Las variables predictoras del modelo fueron: edad, sexo, diagnóstico: IAMNST o AI; cambios del segmento ST, biomarcadores positivos, acceso vascular: radial y/o femoral; fracción de expulsión, creatinina y factores de riesgo como hipertensión arterial, tabaquismo, diabetes mellitus, dislipidemia, antecedente personal y familiar de enfermedad coronaria.

\section{Selección de pacientes}

Se incluyeron pacientes mayores de 18 años, en quienes se establecía el diagnóstico de SCA sin elevación del segmento ST con base en los siguientes criterios: 1) IAMNST, definido como aquel que tiene clínica compatible y/o cambios electrocardiográficos y elevación de troponina por encima del percentil 99, pero no cumple con los criterios electrocardiográficos de infarto agudo del miocardio con elevación del ST; 2) AI, definida por cuadro clínico compatible, ausencia de elevación de troponina, con angina de al menos 10 minutos y que ocurrió en las 24 horas previas al ingreso y que se acompaña de evidencia de enfermedad cardiaca isquémica evidenciada por uno de tres criterios: a. Una nueva depresión del ST de al menos $0.1 \mathrm{mV}$, elevación transitoria del ST o cambios en la $\mathrm{T}$ en al menos dos derivadas contiguas; $b$. Infarto del miocardio previo documentado o procedimiento de revascularización, o c. Resultado de una prueba invasiva o no invasiva que sugiere enfermedad isquémica miocárdica.

Los criterios de exclusión fueron pacientes a quienes por alguna razón de tipo logística no se les podía hacer seguimiento, pacientes que no aceptaran participar en el seguimiento, bloqueo de rama izquierda nuevo o presumiblemente nuevo, pacientes con marcapasos, elevación persistente del segmento ST, angina secundaria con precipitante establecido tal como falla cardiaca, taquiarritmia o anemia, entre otros; o pacientes revascularizados en forma percutánea o quirúrgica en el mes previo.

Una vez definido que un paciente cumplía los criterios para participar en el estudio, se obtenía la información necesaria, la cual se recolectó en una base de datos diseñada para tal fin, mediante entrevista al paciente y revisión de la historia clínica electrónica y controles ambulatorios por consulta externa de cardiología intervencionista, o vía telefónica en los pacientes que por diversas razones no podían asistir a los controles, hasta un mes de seguimiento posterior al evento, obteniéndose información sobre los eventos incluidos en el desenlace evaluado.

\section{Análisis estadístico}

Se utilizó el programa STATA 12 para el análisis de los datos. Se realizó un análisis univariado con medias de tendencia central y de dispersión para variables cuantitativas y de frecuencias para las cualitativas. Además se confirmó la distribución normal por histograma con gráfico de normalidad; las variables con distribución diferente a la normal se confirmaron con prueba de Shapiro-Wilks, considerando significativa una $\mathrm{p}>0.05$, resultando la creatinina y la fracción de eyección con distribución no normal.

Se realizó además un análisis multivariado, al hacer la comparación de medias para dos grupos independientes en las variables con distribución normal con T de student. Para variables con distribución no normal se utilizó comparación de medianas para dos grupos independientes con prueba no paramétrica de Mann-Withney y para variables categóricas tablas de contingencia 2 x 2 con chi-cuadrado.

Se hizo también evaluación de linealidad por coeficiente de correlación de Pearson o Spearman, por medio de matriz de covariables y métodos para determinar colinealidad como el factor de inflación de la varianza $($ FIV < 10) y el índice de tolerancia $(>0.1)$, y una regresión logística paso a paso hacia atrás (backwardstepwise) para introducir las variables candidatas del modelo explicativo con $\mathrm{p}<0.25$.

La evaluación de la capacidad predictiva del modelo de acuerdo con la bondad de ajuste con prueba de HosmerLemershow, considerando significativa una $\mathrm{p}>0.05$, con chi-cuadrado con 8 grados de libertad (GL). Se evaluaron de igual forma los datos que influencian el modelo y los residuales.

Para la evaluación de la capacidad discriminativa del modelo con la obtención de las características operativas de la prueba bajo una curva ROC y comparación de éstas, además de validación interna del modelo por técnica boostrap con repeticiones de 50 .

Para la comparación de las curvas ROC arrojadas por los dos modelos se utilizó un test de igualdad de las áreas bajo la curva con el algoritmo de DeLong, DeLong y Pearson, con estadístico chi-cuadrado y p $<0.05$ (21). 


\section{Tamaño de la muestra}

El tamaño de la muestra se calculó teniendo en cuenta mínimo cinco desenlaces por cada variable independiente introducida en el modelo, en este caso 35 para la escala TIMI ( 7 variables), 40 para el GRACE (8 variables), por lo que se utiliza el mayor número $(22,23)$. Los desenlaces primarios considerados son reinfarto no fatal, muerte y sangrado en los primeros 30 días, con una frecuencia esperada de eventos del $10 \%$.

\section{Resultados}

El periodo de estudió fue desde el 1 de enero de 2011 hasta el 31 de diciembre de 2013, en el que se recolectaron datos de 422 pacientes que ingresaron con impresión diagnóstica de SCA sin elevación del segmento ST y tuvieron seguimiento al menos durante el primer mes posterior al evento. El análisis de base de datos no demostró problemas con datos perdidos (missing values), los cuales fueron $<$ del $5 \%$ en la variable de fracción de eyección, dado que algunos pacientes fallecieron antes de realizársele ecocardiografía; se hizo imputación de datos por la media cuando era adecuado. El promedio de edad fue de $64.36 \pm 11.9$ años, el $54.1 \%$ fueron hombres y la mayoría ingresaron con diagnóstico de infarto sin elevación del segmento ST (52.7\%) (Tablas 1 y 2).

La fracción de eyección y los niveles de creatinina, tienen distribución no normal (Shapiro Wilks con $\mathrm{p}<0.05$ ). La mayoría de los pacientes ingresaron en Killip I $(90.8 \%)$ y el acceso vascular para cateterismo fue radial en $57.7 \%$, aunque

Tabla 1. Características basales de los pacientes.

\begin{tabular}{|c|c|c|}
\hline Variable & Frecuencia (n) & Porcentaje (\%) \\
\hline \multicolumn{3}{|l|}{ Sexo } \\
\hline Mujer & 193 & 45.6 \\
\hline Hombre & 229 & 54.1 \\
\hline \multicolumn{3}{|l|}{ Diagnóstico } \\
\hline AI & 199 & 47 \\
\hline IAMNST & 223 & 52.7 \\
\hline Hipertensión arterial & 353 & 83.5 \\
\hline Diabetes mellitus & 125 & 29.6 \\
\hline Dislipidemia & 233 & 55.2 \\
\hline Tabaquismo activo & 157 & 37.1 \\
\hline AF enfermedad coronaria & 50 & 11.8 \\
\hline AP enfermedad coronaria & 150 & 35.3 \\
\hline Cambios en el ST & 109 & 25.8 \\
\hline Troponina positiva & 223 & 52.7 \\
\hline Killip I & 384 & 90.8 \\
\hline \multicolumn{3}{|l|}{ Acceso } \\
\hline Radial & 244 & 57.7 \\
\hline Femoral & 178 & 42.1 \\
\hline $\begin{array}{l}\text { AI: Angina inestable, IAM } \\
\text { ST, AF: Antecedente fami }\end{array}$ & $\begin{array}{l}\text { o del miocardio sir } \\
\text { nte personal. }\end{array}$ & ción del segmento \\
\hline
\end{tabular}

en el tiempo, la proporción incrementó, convirtiéndose en el acceso de elección.

Se realizó comparación de medias entre dos grupos de acuerdo con el desenlace compuesto de muerte, ECV, reinfarto y sangrado a 30 días con prueba de $t$ de student para las variables con distribución normal, con prueba no paramétrica se establecieron diferencias de medianas para las variables fracción de eyección y creatinina, y por tablas de contingencia $2 \times 2$ para las variables categóricas con prueba de chi-cuadrado (Tablas 3 y 4). Se encontraron diferencias significativas en cuanto a sexo, diagnóstico de IAMNST, cambios del segmento ST, biomarcadores positivos, clasificación Killip, edad, escala TIMI, escala GRACE y fracción de expulsión. La clasificación de Killip se recategorizó para evitar celdas con 0 de valores observados, con resultados similares.

Se encontró relación lineal por matriz de correlación (Pearson y Spearman) mayor de 0.3 entre la escala GRACE y la edad (0.65), cambios en el segmento ST (0.44), biomarcadores positivos (0.321), clasificación Killip (0.344) y escala de TIMI (0.301), resultado esperado dado que las escalas tienen incluidas esas variables, por la cual no se utilizaron para el análisis de regresión logística por inflar la varianza (VIF>10) e introducir sobreajuste. Existe correlación lineal entre el logit de la variable dependiente y las variables independientes analizadas (lowess smoother).

En el análisis univariado se encontraron asociaciones significativas en todas las variables, excepto en el tipo de acceso vascular, OR: 1.06 (IC 95\% 0.56-2; $\mathrm{p}=0.857$ ) y la fracción de eyección, OR: 0.98 (IC 95\% 0.96-1; $\mathrm{p}=0.057$ ); sin embargo, en el multivariado sólo persiste con significancia estadística la edad, OR: 1.06 (IC 95\% 1.02-1.11; p: 0.002) (Tabla 5).

Se realizó un análisis de regresión logística para construir un modelo predictivo con el método paso a paso (backwardstepwise), incluyendo las variables con $p<0.25$, evitando las que demostraron colinealidad. Se construyeron y compararon los modelos así: 1. Modelo completo GRACE: fracción de eyección y escala GRACE, 2. Modelo completo TIMI: fracción de eyección, creatinina y escala TIMI y dos modelos con las escalas solas, 3 . Modelo nulo: GRACE y 4: Modelo nulo: TIMI. El logaritmo negativo de

Tabla 2. Variables cuantitativas basales de los pacientes.

\begin{tabular}{|l|c|c|}
\hline Variable & Media mediana & Desviación típica \\
\hline Edad (años) & 64.36 & 11.9 \\
\hline Fracción de expulsión \% & $55^{*}$ & 15 \\
\hline TIMI & 3.06 & 1.42 \\
\hline Frecuencia cardiaca & 76.5 & 16.7 \\
\hline PAS mmHg & 133.7 & 26.37 \\
\hline Creatinina mg/dL & $1 *$ & 1.66 \\
\hline GRACE & 120.82 & 36.362 \\
\hline *Distribuciones no normales. PAS: Presión arterial sistólica \\
\hline
\end{tabular}


Tabla 3. Comparación de variables predictoras continuas y discretas en cuanto al desenlace compuesto a 30 días (chi-cuadrado).

\begin{tabular}{|c|c|c|c|c|c|}
\hline \multirow[t]{2}{*}{ Variable } & \multicolumn{2}{|c|}{ Desenlace compuesto } & \multirow[t]{2}{*}{ Total } & \multirow[t]{2}{*}{ Chi-cuadrado } & \multirow[t]{2}{*}{$\mathbf{P}$} \\
\hline & $\begin{array}{l}\text { Negativo } \\
1 \text { mes (n) }\end{array}$ & $\begin{array}{c}\text { Positivo } \\
1 \text { mes (n) }\end{array}$ & & & \\
\hline \multicolumn{6}{|l|}{ Sexo } \\
\hline Mujer & 166 & 27 & 103 & 4.835 & 0.02 \\
\hline Hombre & 212 & 17 & 229 & & \\
\hline \multicolumn{6}{|l|}{ Diagnóstico } \\
\hline $\mathrm{AI}$ & 186 & 13 & 199 & 6.114 & 0.013 \\
\hline IAMNST & 192 & 31 & 223 & & \\
\hline \multicolumn{6}{|l|}{ Hipertensión } \\
\hline Sí & 313 & 39 & 353 & 0.893 & 0.345 \\
\hline No & 64 & 5 & 69 & & \\
\hline \multicolumn{6}{|l|}{ Diabetes } \\
\hline Sí & 112 & 13 & 125 & 0.0001 & 0.991 \\
\hline No & 266 & 31 & 297 & & \\
\hline \multicolumn{6}{|l|}{ Dislipidemia } \\
\hline Sí & 206 & 27 & 189 & 0.751 & 0.386 \\
\hline No & 172 & 17 & 233 & & \\
\hline \multicolumn{6}{|l|}{ Tabaquismo } \\
\hline $\mathrm{Si}$ & 141 & 16 & 157 & 0.015 & 0.903 \\
\hline No & 237 & 28 & 265 & & \\
\hline \multicolumn{6}{|l|}{ AF EAC } \\
\hline Sí & 42 & 8 & 50 & 1.887 & 0.17 \\
\hline No & 336 & 36 & 372 & & \\
\hline \multicolumn{6}{|l|}{ AP EAC } \\
\hline Sí & 136 & 14 & 150 & 0.298 & 0.585 \\
\hline No & 242 & 30 & 272 & & \\
\hline \multicolumn{6}{|l|}{ Cambios ST } \\
\hline Sí & 91 & 18 & 109 & 5.831 & 0.016 \\
\hline No & 287 & 26 & 313 & & \\
\hline \multicolumn{6}{|c|}{ Biomarcadores positivos } \\
\hline Sí & 180 & 29 & 209 & 5.274 & 0.022 \\
\hline No & 198 & 15 & 213 & & \\
\hline \multicolumn{6}{|l|}{ Acceso } \\
\hline Radial & 218 & 26 & 244 & 0.033 & 0.857 \\
\hline Femoral & 160 & 18 & 178 & & \\
\hline \multicolumn{6}{|l|}{ Killip } \\
\hline I & 348 & 36 & 384 & & \\
\hline II & 14 & 6 & 20 & 8.965 & 0.030 \\
\hline III & 14 & 2 & 16 & & \\
\hline IV & 2 & 0 & 2 & & \\
\hline
\end{tabular}

la máxima verosimilitud y la seudoR ${ }^{2}$ de Nagelkerke en el modelo inicial con las variables y las escalas demuestran mejor poder predictivo para el modelo completo; sin embargo, la diferencia no es significativa y teniendo en cuenta el principio de parsimonia se pueden escoger los modelos con solo la inclusión de las escalas (modelo nulo), máxime que su operativización es simple. La calibración de los dos modelos finales de acuerdo con el estadístico de HosmerLemershow arrojó chi-cuadrado de 1.39 con p de 0.96 para la escala TIMI y 114 y 0.86 para la escala GRACE (Tabla 6). No se encontraron influencias importantes de observaciones individuales ni con el análisis de los residuales (Leverage, devianza y BIC).

La discriminación de los dos modelos es adecuada con estadístico C de 0.65 para TIMI y 0.69 para GRACE. La comparación de las dos curvas ROC no demuestra diferencias estadísticamente significativas $(\mathrm{p}=0.39)$ (Figura 1).

Con la técnica de boostrap con repeticiones de 50 no hay variaciones significativas de los coeficientes (TIMI coeficiente B 0.397, bootstrap IC 95\% 0.179-0.661; GRACE coeficiente B 0.016, bootstrap 0.01-0.025), demostrando adecuada validación interna de ambos modelos. 
Tabla 4. Comparación de variables predictoras continuas y discretas en cuanto al desenlace compuesto a 30 días.

\begin{tabular}{|c|c|c|c|c|c|}
\hline \multirow[t]{2}{*}{ Variable } & \multicolumn{2}{|c|}{ Desenlace compuesto } & \multirow[t]{2}{*}{ Total } & \multirow[t]{2}{*}{ t Mann -Withney* } & \multirow[t]{2}{*}{$\mathbf{P}$} \\
\hline & $\begin{array}{l}\text { Negativo } \\
1 \text { mes (n) }\end{array}$ & $\begin{array}{l}\text { Positivo } \\
1 \text { mes (n) }\end{array}$ & & & \\
\hline Edad & $63.45 \pm 11.73$ & $72.20 \pm 10.29$ & 422 & 4.74 & 0.0000 \\
\hline TIMI & $2.98 \pm 1.41$ & $3.77 \pm 1.37$ & 422 & 3.53 & 0.0000 \\
\hline Frecuencia cardiaca & $76.24 \pm 16.85$ & $78.50 \pm 15.18$ & 422 & 0.85 & 0.396 \\
\hline PAS & $133.6 \pm 26.55$ & $134 \pm 25$ & 422 & 0.098 & 0.922 \\
\hline GRACE & $118.38 \pm 36$ & $141 \pm 32.52$ & 422 & 4.105 & 0.0000 \\
\hline Creatinina & $1^{*}$ & & 422 & $6.161^{*}$ & 0.079 \\
\hline $\mathrm{FE}$ & $55^{*}$ & & 422 & $6.409 *$ & 0.023 \\
\hline
\end{tabular}

Tabla 5. Análisis univariado y multivariado.

\begin{tabular}{|c|c|c|c|c|c|c|}
\hline \multirow[t]{2}{*}{ Variable } & \multicolumn{3}{|c|}{ Univariado } & \multicolumn{3}{|c|}{ Multivariado } \\
\hline & OR & IC $95 \%$ & $\mathbf{P}$ & OR & IC $95 \%$ & $\mathbf{P}$ \\
\hline Sexo & 2 & $1.07-3.8$ & 0.03 & 1.74 & $0.87-3.48$ & 0.11 \\
\hline Diagnóstico & 2.3 & $1.17-4.55$ & 0.016 & 1.87 & $0.66-5.32$ & 0.23 \\
\hline Cambios ST & 2.18 & $1.14-4.16$ & 0.018 & 1.65 & $0.81-1.41$ & 0.16 \\
\hline Biomarcador & 2.12 & $1.1-4.09$ & 0.024 & 1.11 & $0.39-3.11$ & 0.84 \\
\hline Acceso & 1.06 & $0.56-2$ & 0.857 & 1.26 & $0.63-2.52$ & 0.5 \\
\hline Edad & 1.07 & $1.03-1.1$ & 0.000 & 1.06 & $1.02-1.11$ & 0.002 \\
\hline TIMI & 1.5 & $1.18-1.86$ & 0.01 & 1.21 & $0.93-1.57$ & 0.152 \\
\hline GRACE & 1.48 & $1.18-1.86$ & 0.001 & 0.99 & $0.98-1.01$ & 0.63 \\
\hline Fracción de expulsión & 0.98 & $0.96-1.00$ & 0.057 & 0.97 & $0.95-1.00$ & 0.069 \\
\hline
\end{tabular}

Tabla 6. Modelos comparados en el análisis de regresión logística.

\begin{tabular}{|c|c|c|c|c|}
\hline Característica & Coeficiente & $\mathbf{P}$ & Devianza & BIC \\
\hline \multicolumn{5}{|l|}{ TIMI } \\
\hline Modelo nulo TIMI & 0.39731 & 0.001 & 269 & -2268 \\
\hline Modelo completo TIMI & 0.42931 & 0.001 & 219 & -1657 \\
\hline Fracción expulsión & 0.00394 & 0.142 & & \\
\hline Creatinina & 0.07948 & 0.322 & & \\
\hline \multicolumn{5}{|l|}{ GRACE } \\
\hline Modelo nulo GRACE & 0.01640 & 0.000 & 266 & -2272 \\
\hline Modelo completo GRACE & 0.01520 & 0.001 & 220 & -1656 \\
\hline Fracción expulsión & 0.00371 & 0.203 & & \\
\hline Creatinina & 0.07553 & 0.350 & & \\
\hline
\end{tabular}

\section{Discusión}

El pronóstico de pacientes con SCA sin ST es bastante heterogéneo (24); sin embargo, se ha logrado determinar algunas variables pronósticas importantes que discriminan adecuadamente el riesgo, entre las que se encuentran la edad, el sexo, el tabaquismo, la historia de diabetes, angina previa o falla cardiaca, la revascularización percutánea o quirúrgica previa y algunas variables hemodinámicas y de laboratorio, como la frecuencia cardiaca, la fracción de eyección, la presión arterial y la disfunción renal (25).

La construcción de modelos multivariables de predicción de riesgo, surgieron como una necesidad para identificar 


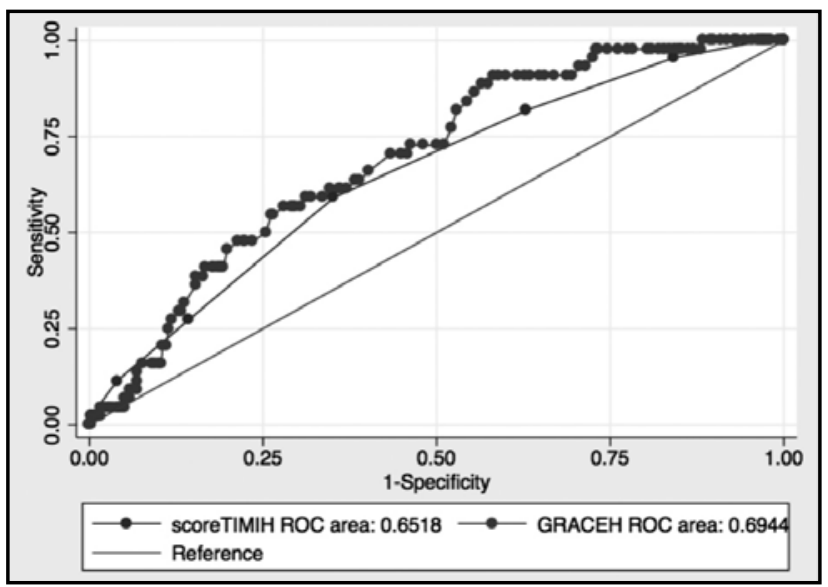

Figura 1. Curvas ROC de modelos con GRACE y TIMI y su comparación.

pacientes de alto riesgo que se benefician de terapias agresivas y estrategia de estratificación invasiva, en los cuales se puede minimizar el riesgo de eventos adversos al utilizarse en pacientes con mayor tasa de desenlaces. Los más utilizados y validados prospectivamente son el TIMI y el GRACE.

Reportamos los resultados del análisis de una población contemporánea de pacientes con SCA sin elevación del segmento ST, tratados en dos unidades especializadas, con tratamiento de acuerdo con las guías de práctica clínica de Colombia (26) y alta tasa de intervencionismo percutáneo, específicamente en pacientes de alto riesgo (datos no mostrados). Los datos de seguimiento a corto plazo demuestran una tasa elevada de eventos $(10.43 \%)$, confirmando que es una población de alto riesgo. El análisis univariado permitió identificar algunas variables relacionadas con el desenlace compuesto de muerte, reinfarto, ECV y sangrado a 30 días, tales como sexo masculino, IAMNST, cambios electrocardiográficos iniciales, biomarcadores positivos y la edad; sin embargo, sólo esta última persiste significativa en el análisis multivariado. Este análisis permitió la construcción de un modelo de predicción que incluye tres variables. Las variables incluidas fueron: fracción de expulsión, creatinina y la escala TIMI o fracción de expulsión y la escala GRACE. Hay que tener en cuenta que se incluyeron como variables en los modelos la fracción de expulsión y la creatinina con el TIMI y solamente fracción de expulsión con el GRACE, debido a que en estudios previos han demostrado ser marcadores pronósticos independientes, que pueden aportar mejor poder de predicción a las escalas solas $(27,28)$. Las escalas se analizaron en forma separada, dado que comparten variables similares en la predicción del riesgo, y a su vez se compararon con el modelo con tres variables, mencionadas como modelos completos, bien sea TIMI o GRACE según la escala utilizada. Los dos modelos están bien calibrados dados los resultados de la prueba de bondad de ajuste de Hosmer-Lemeshow y el estadístico chi-cuadrado de Pearson, adicionalmente con el análisis de residuales, la "devianza" y el criterio de información Bayesiano (BIC).

Aspectos importantes por considerar en la utilización de estos puntajes son: primero que la población incluida represente en forma adecuada a la población general, y segundo que el modelo proporcione predicciones precisas del riesgo. Para esto se utiliza el estadístico C, el cual es la proporción de pares de pacientes, uno sin el desenlace y otro con el desenlace, en el cual el paciente con el desenlace tiene la más alta probabilidad predicha del evento. Un modelo que exactamente discrimine los pacientes $85 \%$ de las veces tendrá un estadístico $\mathrm{C}$ de 0.85 ; predicciones aleatorias como al lanzar una moneda, tendrá un estadístico $\mathrm{C}$ de 0.50 . Se considera entonces, que estadísticos $\mathrm{C}$ de 0.6 o menos no tiene valor clínico, de 0.6 - 0.7 tiene valor limitado, de 0.7 - 0.8 tiene un valor modesto y mayor de 0.8 tiene un valor clínico importante $(29,30)$.

En general los dos modelos finales, que sólo incluyen la escala, tienen una capacidad discriminatoria adecuada con estadístico C de 0.65 para la escala TIMI y 0.69 para la escala GRACE, que a simple vista podría refrendar la mejor capacidad reportada para esta última (31); sin embargo, la comparación no demuestra diferencia estadísticamente significativa; esto puede ser debido a que el GRACE tiene mejor poder de predicción para el desenlace aislado de muerte y no para el compuesto (32) y aun mejor para el seguimiento a los seis meses.

Los resultados muestran que los modelos con dos o tres variables (modelo completo) tienen mejor ajuste, sin embargo en aras de simplificar y de la operativización, con la premisa de la parsimonia, los modelos con solo la escala (modelo nulo) son suficientes.

Nuestros datos están de acuerdo con estudios similares de validación de las dos escalas mencionadas en diferentes poblaciones (33-35).La discriminación de la escala GRACE por el estadístico $\mathrm{C}$ para mortalidad e infarto fue de $0.81 \mathrm{y}$ 0.73 y un reciente metaanálisis que incluye 22 estudios de validación, que encontró un estadístico $\mathrm{C} \geq \mathrm{a} 0.80$ en ambos tipos de SCA a corto y largo plazo. A pesar de esto, el registro GRACE sólo incluyó un $26.6 \%$ de pacientes intervenidos en forma percutánea y es en ese grupo de pacientes donde tiene menor poder (estadístico C de 0.73); sin embargo, en cohortes contemporáneas de tratamiento, en las cuales la realización de revascularización miocárdica es mayor, no se encuentran diferencias significativas entre la población sometida a estos procedimientos en comparación a los que se les da manejo medico (Estadístico C 0.835 revascularización percutánea vs 0.906 en manejo médico) (32).

La discriminación de la escala TIMI en el modelo de derivación y validación, tuvo un estadístico $\mathrm{C}$ de 0.59 y 0.65 , con aumento significativos en las tasas de eventos. El modelo fue modestamente predictivo para el desenlace final compuesto de muerte, infarto no fatal e isquemia recurrente severa que requiere revascularización urgente (estadístico C 0.63), con mejor predicción para mortalidad (estadístico C 
0.72-0.78); el modelo sacrifica poder de predicción al excluir la presencia de falla cardiaca y variables hemodinámicas en aras de su simplicidad $(37,38)$.

Los dos modelos anteriores son utilizados rutinariamente en los servicios de urgencias para determinar el subgrupo de pacientes con IAMNST/AI de más alto riesgo, a quienes se les debe realizar intervención coronaria percutánea como medida terapéutica específica inicial. Son sencillos, requieren pocos parámetros de laboratorio y se pueden efectuar en toda institución prestadora de salud de segundo nivel o superior. Sin embargo, no todas las cohortes a nivel internacional han obtenido resultados similares con la aplicación de estas escalas (39). Diferencias sociodemográficas, étnicas, genéticas o idiosincráticas son causales de que los resultados predichos en los estudios iniciales no se hayan reproducido con exactitud en poblaciones diferentes a las originales en las cuales se diseñaron (40). Es así como en Latinoamérica se han realizado estudios evaluando estas escalas con resultados variables. Bagur y colaboradores en Argentina (34), evaluaron en 204 pacientes, los eventos cardiovasculares a seis meses de seguimiento con el puntaje de riesgo TIMI, encontrando que aquellos con puntajes en riesgo intermedio o alto, se correlacionaron con nuevos eventos cardiovasculares. Por otro lado, Correia y colaboradores (35), evaluaron en Brasil a 154 pacientes con SCA sin elevación del ST, encontraron que la escala GRACE tenía capacidad pronóstica superior al TIMI en cuanto a la predicción de eventos hospitalarios. Los resultados han sido replicados en una cohorte contemporánea de pacientes con SCA en España, encontrando adecuada reproducibilidad para predecir mortalidad a los seis meses (32).

En nuestro país, Ocampo y colaboradores (41) realizaron también un análisis sobre la exactitud pronóstica de las escalas de TIMI y GRACE, encontrando bajos niveles de predicción; sin embargo, no describen la metodología de construcción del modelo (42) y el resultado de la curva ROC menor de 0.5 para la escala TIMI sugiere problemas en la codificación del desenlace; mientras los resultados de nuestro modelo de predicción, permiten dar unos valores pronósticos aceptables para ambas escalas.

Las limitaciones del estudio están relacionadas con el modesto tamaño de la muestra, que aunque cumple con la estipulada para el tipo de estudio, limita la inclusión de un mayor número de variables, dada la tasa de desenlaces, que obliga a utilizar uno compuesto. A este respecto se debe enfatizar que la escala GRACE tiene mejor predicción para mortalidad. La utilización de cohortes retrospectivas es otra de las limitaciones, aunque aceptada internacionalmente. De igual forma también debe explorarse la inclusión de nuevos marcadores (péptido natriurético, entre otros) con la intención de establecer el índice neto de reclasificación (NRI), para mejor discriminación, adicional al estadístico C. El uso de técnicas de validación interna es importante; sin embargo, al ejecutarse con la misma muestra puede generar dificultades, por lo que debe realizarse en poblaciones diferentes, con el objetivo de verificar la validez externa y por lo tanto reproducibilidad.

\section{Conclusión}

En pacientes con síndrome coronario agudo sin elevación del segmento ST mayores de 18 años, evaluados en unidades especializadas con disponibilidad de laboratorio de hemodinámica, la realización de un modelo de regresión logística mostró mejor poder predictivo para el uso de tres variables; sin embargo, esto no tuvo una diferencia estadísticamente significativa con la realización de sólo las escalas de TIMI o GRACE, las cuales tuvieron una buena capacidad de predicción del compuesto de muerte, reinfarto, ECV y sangrado a 30 días, en pacientes mayores de 18 años con SCA sin elevación del segmento ST.

\section{Declaración de fuentes de financiación y posibles conflictos de interés}

El presente estudio fue financiado con recursos propios de la Sección de Cardiología de la Facultad de Medicina de la Universidad de Antioquia y del grupo para el estudio de las enfermedades cardiovasculares.

No hay conflictos de interés.

\section{Referencias}

1. Steg HG, James SK, Atar D et al. ESC Guidelines for the management of acute myocardial infarction in patients presenting with ST-segment elevation. Eur Heart J 2012; 33: 2569-2619.

2. O'Gara PT, Kushner FG, Ascheim DD. 2013 ACCF/AHA Guideline for the Management of ST-Elevation Myocardial Infarction. Circulation 2013; 127:529555 .

3. Cantor WJ, Fitchett D, Borgundvaag B et al. Routine Early Angioplasty after Fibrinolysis for Acute Myocardial Infarction. N Engl J Med 2009; 360:2705-18.

4. Bøhmer E, Hoffmann P, Abdelnoor $\mathbf{M}$ et al. Efficacy and Safety of Immediate Angioplasty Versus Ischemia-Guided Management After Thrombolysis in Acute Myocardial Infarction in Areas With Very Long Transfer Distances. J Am Coll Cardiol 2010;55:102-10

5. Swap CJ, Nagurney JT. Value and Limitations of Chest Pain History in the Evaluation of Patients With Suspected Acute Coronary Syndrome. JAMA 2005; 294: 2623-2629.

6. Nyman I, Areskog M, Areskog NH, Swahn E, Wallentin L. Very early risk stratification by electrocardiogram at rest in men with suspected unstable coronary heart disease. The RISC Study Group. J Intern Med 1993; 234:293-301.

7. Cannon CP, McCabe CH, Stone PH et al. The Electrocardiogram Predicts OneYear Outcome of Patients With Unstable Angina and Non-Q Wave Myocardial Infarction: Results of the TIMI III Registry ECG Ancillary Study. J Am Coll Cardiol 1997;30:133-40.

8. Savonnito S, Ardissino D, Granger CB et al. Prognostic value of the admission electrocardiogram in acute coronary syndromes. JAMA 1999; 281:707-713.

9. Antman E, Tanasijevic I, Thompson B et al. Cardiac Specific Troponin I Levels to Predict the Risk of Mortality in Patients with Acute Coronary Syndromes. $N$ Engl J Med 1996;335:1342-9.

10. Ohman M, Armstrong P, Christenson R et al. Cardiac Troponin T Levels for Risk Stratification in Acute Myocardial Ischemia. N Engl J Med 1996; 335:133341 .

11. Reichlin T, Hochholzer W, Bassetti S et al. Early Diagnosis of Myocardial Infarction with Sensitive Cardiac Troponin Assays. N Engl J Med 2009; 361:858-67.

12. Amsterdam EA, Wenger NK, Brindis RG, Casey DE, Ganiats TG et al. 2014 AHA/ACC Guideline for the Management of Patients with Non-ST-Elevation Acute Coronary Syndromes: a report of the American College of Cardiology/ American eart Association Task Force on Practice Guidelines. Circulation. 2007 Ago;116(7):e148-e304.

13. Boersma E, Pieper KS, Steyerberg EW et al. For the PURSUIT Investigators Predictors of outcome in patients with acute coronary syndromes with out persistent ST-segment elevation: results from an international trial of 9461 patients. Circulation 2000;101:2557-67.

14. Lagerqvist B, iderholm E, Lindahl B et al. FRISC score for selection of patients 
for anearly invasive treatment strategy in unstable coronary artery disease. Heart 2005;91:1047-1052.

15. Backus BE, Six AJ, Kelder JC. Chest Pain in the Emergency Room A Multicenter Validation of the HEART Score. Crit Pathways in Cardiol 2010;9: 164-169.

16. Antman EM, Cohen M, Bernink PJ, et al. The TIMI Risk Score for Unstable Angina/Non-ST Elevation MI: A Method for Prognostication and Therapeutic Decision Making. JAMA 2000;16:835-42.

17. Morrow DA, Antman EM, Charlesworth A et al. TIMI Risk Score for STElevation Myocardial Infarction: A Convenient, Bedside, Clinical Score for Risk Assessment at Presentation. Circulation 2000;102:2031-2037.

18. Granger C, Goldberg RJ, Dabbous $\mathbf{O}$ et al. Predictors of hospital mortality in the global registry of acute coronary events. Arch Intern Med 2003; 163:2345-2353.

19. Eagle KA, Lim MJ, Dabbous OH, et al. A validated prediction model for all forms of acute coronary syndrome: estimating the risk of 6 - month post discharge death in an international registry. JAMA 2004;291:2727-33.

20. Fox K, Dabbous O, Goldberg RJ et al. Prediction of risk of death and myocardial infarction in the six month safter presentation with acute coronary syndrome: prospective multinational observational study (GRACE). BMJ 2006; 333:1091-4.

21. De Long E, De Long D, Pearson DLC. Comparing the areas under two or more correlated receiver operating characteristic curves: a non parametric approach. Biometrics 1988; 44: 837-845.

22. Vittinghoff $\mathbf{E}$, McCulloch. Relaxing the rule events per variable in logistic and cox regression. Am J Epidemiol 2007; 165: 710-718.

23. Vergouwe Y, Steyeberg E, Eijkemans M, Habbena JDF. Substantial effective sample sizes were required for external validation studies of predictive logistic regression models. J Clin Epidemiol 2005; 58: 475-483.

24. Kumbhani D, Wells BJ , Lincoff AM et al. Predictive models for short- and long-term adverse out comes following discharge in a contemporary population with acute coronary sindromes. Am J Cardiovasc Dis 2013;3:39-52.

25. Mahaffey KW, Yang Q, Pieper KS et al. Prediction of One-Year Survival in High-Risk Patients with Acute Coronary Syndromes: Results from the SYNERGY Trial. J Gen Intern Med. 2008 Mar;23(3):310-6.

26. Senior JM, Lugo LH, Acosta N et al. Guía de práctica clínica para pacientes con diagnóstico de síndrome coronario agudo: atención inicial y revascularización. Rev Colomb Cardiol 2013;20 (Suplemento 2):45-85

27. Bosch X, Théroux P. Left ventricular ejection fraction to predict early mortality in patients with non-ST-segment elevation acute coronary syndromes. Am Heart $J$ 2005;150:215-20.

28. Santopinto JJ, Fox KA, Goldberg RJ et al. Creatinine clearance and adverse hospital outcomes in patients with acute coronary syndromes: findings from the global registry of acute coronary events (GRACE). Heart 2003;89:1003-08.
29. Harrell E Jr, Califf R, Pryor DB, Lee KL, Rosati RA. Evaluating the yield of medical tests. JAMA. 1982;247:2543-2546.

30. Ohman E, Magnus MD, Granger C et al. Risk Stratification and Therapeutic Decision Making in Acute Coronary Syndromes. JAMA. 2000;284:876-878.

31. Aragam K, Tamhane U, Kline-Rogers E, et al. Does Simplicity Compromise Accuracy in ACS Risk Prediction? A Retrospective Analysis of the TIMI and GRACE Risk Scores. PLOS ONE 2009; 11: e7947.

32. Abu-Assi E, García-Acuña JM, Peña-Gil C y González-Juanatey JR. Validación en una cohorte contemporánea de pacientes con síndrome coronario agudo del score GRACE predictor de mortalidad a los 6 meses de seguimiento. Rev Esp Cardiol. 2010;63:640-8.

33. Ohman E, Magnus MD, Granger C, et al. Risk Stratification and Therapeutic Decision Making in Acute Coronary Syndromes. JAMA. 2000;284:876-878.

34. Bagur RH, Urinovsky F, Contreras A, Estrada CD. Validación del score de riesgo TIMI para pacientes con síndrome coronario agudo sin elevación del ST. MEDICINA (Buenos Aires) 2009; 69: 526-528.

35. Correia LC, Freitas R, Bittencourt AP, et al. Prognostic value of GRACE scores versus TIMI score in acute coronary syndromes. Arq Bras Cardiol 2010;94(5):6139.

36. D'Ascenzo F, Biondi-Zoccai G, Moretti C, et al. TIMI, GRACE and alternative risk scores in acute coronary síndromes: A meta-analysis of 40 derivations studies on 216552 patients and of 42 validation studies on 31625 patients. Contemp Clinical Trials 2012;33:507-514.

37. Aragam K, Tamhane U, Kline-Rogers E, et al. Does Simplicity Compromise Accuracy in ACS Risk Prediction? A Retrospective Analysis of the TIMI and GRACE Risk Scores. PLOS ONE 2009;11: e7947.

38. Yan AT, Yan RT, Tan M, et al. Risk scores for risk stratification in acute coronary syndromes: useful but simpler is not necessarily better. European Heart Journal (2007) 28, 1072-1078.

39. Filipiak K, Koltowski L, Grabowski M et al. Prospective Comparison of the 5 Most Popular Risk Scores in Clinical Use for Unselected Patients With Acute Coronary Syndrome. Circ J 2011; 75: 167 - 173.

40. Chang WCH, Midodzi W, Westerhout C, et al. Are international differences in the outcomes of acute coronary syndromes apparent or real? A multi level analysis. J Epidemiol Community Health 2005;59:427-433.

41. Ocampo LA, Saldarriaga C, Gómez A, et al. Exactitud pronóstica de las escalas GRACE y TIMI en pacientes llevados a intervencionismo percutáneo por síndrome coronario agudo sin elevación del ST. Rev Col Cardiol 2013;20:130-135.

42. Bouwmeester W, Zuithoff N, Mallet $\mathbf{S}$, et al. Reporting and methods in clinical prediction research: a systematic review. PLoSMed 2012; 9: 1-12. 